\title{
ASYMPTOTIC FLUX ACROSS HYPERSURFACES FOR DIFFUSION PROCESSES
}

\author{
ANDREA POSILICANO AND STEFANIA UGOLINI
}

\begin{abstract}
We suggest a rigorous definition of the pathwise flux across the boundary of a bounded open set for transient finite energy diffusion processes. The expectation of such a flux has the property of depending only on the current velocity $v$, the nonsymmetric (with respect to time reversibility) part of the drift. In the case where the diffusion has a limiting velocity we define the asymptotic flux across subsets of the sphere of radius $R$, when $R$ tends to infinity, and compute its expectation in terms of $v$.
\end{abstract}

Keywords: diffusion processes, (random) flux across surfaces, current velocity

\section{INTRODUCTION}

In a previous paper [13] the authors gave a pathwise probabilistic versions of the Scattering-into-Cones and Flux-across-Surfaces theorems in Quantum Mechanics and then recovered the known analytical results by taking suitable expectations.

Here we extend the main probabilistic results contained in [13] to a large class of Markovian diffusions with no a priori connection with Quantum Mechanics.

We consider diffusions on $\mathbb{R}^{d}$ which are weak solutions of s.d.e.'s of the form

$$
d X_{t}=b\left(t, X_{t}\right) d t+d W_{t},
$$

where $W_{t}$ is a standard Wiener process and the drift vector field $b_{t}(x) \equiv$ $b(t, x)$ satisfies

$$
\forall T>0, \quad \mathbb{E} \int_{0}^{T}\left\|b\left(t, X_{t}\right)\right\|^{2} d t<+\infty .
$$

The condition (1.2) is a finite energy condition in the sense of Föllmer [6. By Girsanov theory one proves that (1.2) is equivalent to a finite entropy condition: the probabilitity measure describing the weak solution of (1.1) has finite relative entropy with respect to the Wiener measure. By definition of relative entropy, this fact implies absolute 
continuity and therefore the distribution of $X_{t}, t>0$ is absolutely continuous (with respect to Lebesgue measure) with some density function $\rho_{t}$.

A very peculiar consequence of (1.2) is that not only the Markovian property is preserved under time reversal, but also the diffusion one. We will call this property time reversibility of the diffusive character. The class of diffusion processes individuated by this invariance property was firstly proposed by Nelson in 1966 [10] in the framework of Stochastic Mechanics. Such diffusions were then studied by Zheng and Meyer who called them semimartingales dans les deux directions du temps [14. Within this class, Carlen in 1984 2] (see [4] for an alternative proof) solved the existence problem of weak solutions of (1.1) in the case of (unbounded) drift fields satisfying (1.2). Successively, Föllmer [6] gave a very elegant characterization based on the relative entropy approach. Under time reversal the process solution of (1.1) is again a solution of a s.d.e. of type (1.1) with some dual drift field $b_{t}^{*}$ (and, of course, another standard Wiener process) which satisfies the relation:

$$
b_{t}(x)-b_{t}^{*}(x)=\nabla \log \rho_{t}(x)
$$

The duality relation (1.3) allows to introduce a relevant decomposition of the drift field as the sum of two vector fields :

$$
v_{t}=\frac{b_{t}+b_{t}^{*}}{2}, \quad u_{t}=\frac{b_{t}-b_{t}^{*}}{2}
$$

called respectively current and osmotic velocity [11. In the symmetric case, $v_{t}=0$ and thus $b_{t}=u_{t}$ is of gradient type according to (1.3). Therefore the current velocity $v_{t}$ represents the non symmetric part of the drift field. We will see that only the current velocity is involved in the expression of the flux across surfaces by the diffusion paths.

A useful consequence of the time reversibility of the diffusion property is then the validity (in the weak sense) of the continuity equation for the couple $(\rho, v)$ :

$$
\frac{\partial}{\partial t} \rho_{t}=-\nabla \cdot\left(\rho_{t} v_{t}\right)
$$

In this paper we give a rigorous definition of the pathwise flux across the boundary of a bounded set by a transient Markov diffusion process solution of (1.1) and satisfying the energy condition (1.2).

Given a bounded open set $D$ let us consider the function

$$
N_{\partial D}:=N_{\partial D}^{+}-N_{\partial D}^{-},
$$

where $N_{\partial D}^{+}(\gamma)$ (resp. $N_{\partial D}^{-}(\gamma)$ ) denotes the number of inward (resp. outward) crossing by the path $t \mapsto X_{t}(\gamma)$ of the boundary $\partial D, \gamma$ being 
the point in the probability space. It is not a local time because we need to distinguish outward from inward crossing in order to have the pathwise analogoue of a net flux. The problem is that almost surely the diffusion $X_{t}$ intersects $\partial D$ on a set of times that has no isolated points and is uncountable. Therefore the definition of $N_{\partial D}$ given above makes no sense in general. However, by a suitable redefinition of $N_{\partial D}$ as the total mass of the almost surely compactly supported random distribution $-\frac{d}{d t} \chi_{D}\left(X_{t}\right)$, where $\chi_{D}$ is the characteristic function of the set $D$ (see section 3 for details), we can give a rigorous definition of the pathwise flux across $\partial D$. Then, by using the continuity equation (1.4), in the case where $\partial D$ is a regular hypersurface we can compute (see theorem 3.2) the expectation $\Phi_{\partial D}$ of the pathwise flux $N_{\partial D}$ in terms of the current velocity $v$, obtaining

$$
\Phi_{\partial D}=\int_{0}^{+\infty} \int_{\partial D} \rho(t, x) v(t, x) \cdot n(x) d \sigma(x) d t,
$$

where $n$ denotes the outward unit normal vector along $\partial D$ and $\sigma$ is the surface measure. We interpret $\Phi_{\partial D}$ as the flux of $X_{t}$ across $\partial D$. Note that, by our choice of signs in (1.5), D is a source if $\Phi_{\partial D}>0$ and is a sink if $\Phi_{\partial D}<0$.

Of course the definition of flux given above does not extend to the case of a hypersurface which is not a boundary. This restriction can be avoided, at least asymptotically, in the case where the diffusion $X_{t}$ has a limiting velocity, i.e.

$$
\lim _{t \uparrow \infty} \frac{1}{t} X_{t}=v_{\infty}
$$

exists almost surely for some non zero random vector $v_{\infty}$. A simple but general condition giving (1.7), which is again a finite entropy condition, was obtained by Carlen [3] (see Theorem 4.3 below).

Suppose that $\Sigma$ is an open subset of the unit sphere with $\partial \Sigma$ a finite union of $C^{1}$ manifolds. In order to define the asymptotic flux across $\Sigma$ we consider the cone $C_{\Sigma}:=\left\{\lambda x \in \mathbb{R}^{d}: x \in \Sigma, \lambda>0\right\}$. Using (1.7) (see section 5 for the details) we can then define $N_{\Sigma}^{a}$, the asymptotic pathwise flux across $\Sigma$, by the limit $R \uparrow \infty$ of the mass $N_{\Sigma_{R}}$ of the almost surely compactly supported random distribution $\frac{d}{d t} \chi_{C \cap B_{R}^{c}}\left(X_{t}\right)$, $B_{R}$ being the closed ball of radius $R$. Note the change of sign in the definition of $N_{\Sigma_{R}}$ with respect to $N_{\partial D}$. This is consistent with the fact that the exterior normal to $\Sigma_{R}:=C_{\Sigma} \cap S_{R}, S_{R}$ being the sphere of radius $R$, coincides with the interior normal to the boundary of $C \cap B_{R}^{c}$. No confusion can arise between the two different definitions since $\Sigma_{R}$ is never the boundary of an open subset of $\mathbb{R}^{d}$. 
We show (see theorem 5.2) that $N_{\Sigma}^{a}$ is well defined since almost surely one has

$$
N_{\Sigma}^{a}:=\lim _{R \uparrow \infty} N_{\Sigma_{R}}=\chi_{C_{\Sigma}}\left(v_{\infty}\right)
$$

Moveover, if as before we define the flux by taking the expectation of the corresponding pathwise object, we have (see theorem 5.3)

$$
\Phi_{\Sigma}^{a}=\lim _{R \uparrow \infty} \int_{0}^{+\infty} \int_{\Sigma_{R}} \rho(t, x) v(t, x) \cdot n(x) d \sigma(x) d t .
$$

The conditions required in order to obtain the stated results are, besides (1.1) and (1.2), that $\rho_{t}$ and $v_{t}$ belong to the Sobolev space $H^{1}\left(\mathbb{R}^{3}\right)$ in order to obtain $(1.6),(1.7)$ to get (1.8), and moreover we assume

$$
\mathbb{E} \int_{0}^{T}\left\|\nabla v\left(t, X_{t}\right)\right\|^{2} d t<+\infty
$$

for some $T>0$, to get (1.9).

Finally let us remark that our definition of a pathwise flux across $\partial D$ is euristically equivalent to the ill-defined Stratonovich stochastic integral

$$
\int_{0}^{+\infty}\left(\int_{\partial D} \delta\left(X_{t}-x\right) n(x) d \sigma(x)\right) \circ d X_{t} .
$$

Indeed, proceeding euristically, one obtains

$$
\begin{aligned}
N_{\partial D} & =\int_{0}^{+\infty}-\frac{d}{d t} \chi_{D}\left(X_{t}\right) d t=-\int_{0}^{+\infty} \frac{d X_{t}}{d t} \cdot \nabla \chi_{D}\left(X_{t}\right) d t \\
& =\int_{0}^{+\infty} \frac{d X_{t}}{d t} \cdot n\left(X_{t}\right) \delta_{\partial D}\left(X_{t}\right) d t \\
& =\int_{0}^{+\infty}\left(\int_{\partial D} \delta\left(X_{t}-x\right) n(x) d \sigma(x)\right) \circ d X_{t} .
\end{aligned}
$$

\section{The ClASS OF Finite ENERGy DIFFusion PROCESSES}

Consider the measurable space $(\Omega, \mathcal{F})$, with $\Omega=C\left(\mathbb{R}_{+} ; \mathbb{R}^{d}\right), \mathcal{F}$ the Borel $\sigma$-algebra, and let $\left(\Omega, \mathcal{F}, \mathcal{F}_{t}, X_{t}\right)$ be the evaluation stochastic process $X_{t}(\gamma):=\gamma(t), \gamma \in \Omega$, with $\mathcal{F}_{t}=\sigma\left(X_{s}, 0 \leq s \leq t\right)$ the natural filtration.

Let us suppose that:

H1) there exists a Borel probability measure $\mathbb{P}$ on $(\Omega, \mathcal{F})$ such that:

- $\left(\Omega, \mathcal{F}, \mathcal{F}_{t}, X_{t}, \mathbb{P}\right)$ is a Markov process; 
- $W_{t}:=X_{t}-X_{0}-\int_{0}^{t} b\left(s, X_{s}\right) d s$ is a $\left(\mathbb{P}, \mathcal{F}_{t}\right)$-Wiener process, i.e. $\mathbb{P}$ is a weak solution of the stochastic differential equation

$$
d X_{t}=b\left(t, X_{t}\right) d t+d W_{t}
$$

with initial distribution $\mu=\mathbb{P} \circ X_{0}^{-1}, \mu \ll \lambda, \lambda$ the Lebesgue measure on $\mathbb{R}^{d}$;

H2) the adapted process $b_{t}$ satisfies

$$
\forall T>0, \quad \mathbb{E} \int_{0}^{T}\left\|b\left(t, X_{t}\right)\right\|^{2} d t<+\infty
$$

where $\mathbb{E}$ denotes the expectation with respect to the measure $\mathbb{P}$.

Remark 2.1. Since

$$
H_{\mathcal{F}_{T}}\left(\mathbb{P}, \mathbb{P}^{W}\right):=\mathbb{E}\left(\left.\log \frac{d \mathbb{P}}{d \mathbb{P} W}\right|_{\mathcal{F}_{T}}\right)=\frac{1}{2} \mathbb{E} \int_{0}^{T}\left\|b\left(t, X_{t}\right)\right\|^{2} d t
$$

where $\mathbb{P}^{W}:=\int \mu(x) \mathbb{P}_{x}^{W}$, with $\mathbb{P}_{x}^{W}$ denoting the Wiener measure starting from $x$, see [6], the finite energy condition (2.2) is equivalent to a finite relative entropy condition. Thus (2.2) implies that $\mathbb{P}$ is absolutely continuous with respect to $\mathbb{P}^{W}$, and so $X_{t}$ admits a density function $\rho_{t}$ for any $t \geq 0$.

As a consequence of $\mathrm{H} 1$ and $\mathrm{H} 2$, the Markovian diffusion $X_{t}$ preserves the diffusion property under time reversal. Indeed by Föllmer $[6$, one has:

Lemma 2.2. Under the hypothesis $H 1, H 2$, defining $\hat{\mathbb{P}}:=\mathbb{P} \circ R$, where $R$ is the pathwise time reversal on $C\left([0, T] ; \mathbb{R}^{d}\right), R(\gamma)(t):=\gamma(T-t)$, there exists an adapted process $\hat{b}_{t}$ such that:

$$
\hat{W}_{t}:=X_{t}-X_{0}-\int_{0}^{t} \hat{b}\left(s, X_{s}\right) d s
$$

is a $\left(\hat{\mathbb{P}}, \mathcal{F}_{t}\right)$-Wiener process.

Remark 2.3. Lemma 2.2 states that the finite energy (entropy) condition (2.2) is a sufficient condition for the time reversibility of the diffusion property. The proof is based on the fact that the finite entropy condition is invariant under time reversal. The extension to the infinite dimensional case is in [7]. Sufficient conditions are also given in 8. Sufficient and necessary conditions for reversibility of diffusion property, in the case of Lipschitz drift fields, are investigated in [9]. 
It is well known that the drift field can be seen as a stochastic forward derivative in the sense of Nelson [10, [11. In particular from (2.1) and (2.2) it follows that (see [6]):

$$
b_{t}=\lim _{h \downarrow 0} \frac{1}{h} \mathbb{E}\left[X_{t+h}-X_{t} \mid \mathcal{F}_{t}\right] \quad \text { in } L^{2}(\mathbb{P}) .
$$

Analogously, from (2.3) one also has:

$$
\hat{b}_{t}=\lim _{h \downarrow 0} \frac{1}{h} \hat{\mathbb{E}}\left[X_{t+h}-X_{t} \mid \mathcal{F}_{t}\right] \quad \text { in } L^{2}(\hat{\mathbb{P}}),
$$

where $\hat{\mathbb{E}}$ denotes the expectation with respect to the measure $\hat{\mathbb{P}}$. For our approach it is convenient to work with the same probability measure $\mathbb{P}$ as proposed by Nelson [10]. To this end we write:

$$
\hat{b}_{t}=\lim _{h \downarrow 0}-\frac{1}{h} \mathbb{E}\left[X_{T-t}-X_{T-t-h} \mid \hat{\mathcal{F}}_{T-t}\right] \circ R \quad \text { in } L^{2}(\mathbb{P}),
$$

where $\hat{\mathcal{F}}_{t}=\sigma\left(X_{s}, s \geq t\right)$ is the natural future filtration.

Since the Markov property is preserved under time reversal also the dual drift is given by some measurable function $\hat{b}_{t}(\gamma)=\hat{b}\left(t,\left(X_{t}(\gamma)\right)\right.$. Let us define $b_{t}^{*}(x)=-\hat{b}_{T-t}(x)$ so that, as already obtained in [11]:

$$
b_{t}^{*}=\lim _{h \downarrow 0}-\frac{1}{h} \mathbb{E}\left[X_{t}-X_{t-h} \mid \hat{\mathcal{F}}_{t}\right] \quad \text { in } L^{2}(\mathbb{P}),
$$

and the following relation holds:

$$
b_{t}(x)-b_{t}^{*}(x)=\nabla \log \rho_{t}(x)
$$

between the drift field and its dual (see [2] and for the non Markovian case [6]).

The duality relation (2.4) allows to introduce the decomposition

$$
b_{t}=u_{t}+v_{t}, \quad b_{t}^{*}=-u_{t}+v_{t}
$$

where

$$
v_{t}=\frac{b_{t}+b_{t}^{*}}{2}, \quad u_{t}=\frac{b_{t}-b_{t}^{*}}{2}
$$

are called current and osmotic velocity respectively [10.

In the symmetric case, $v_{t}=0, b_{t}=u_{t}, b_{t}^{*}=-u_{t}$, thus the drift field coincides up to the sign with its dual and, according to (2.4), it is of gradient type. Therefore the current velocity $v_{t}$ represents the not symmetric (with respect to time reversal) part of the drift field. We will see that only the current velocity is involved in the expression of the flux across surfaces.

An important consequence of the time reversibility of the diffusion property is the validity of the continuity equation (in the weak sense) for the couple $(\rho, v)$. 
Indeed, recalling the Fokker-Planck equation associated with (2.1):

$$
\frac{\partial}{\partial t} \rho_{t}=-\nabla \cdot\left(\rho_{t} b_{t}\right)+\Delta \rho_{t}
$$

and the Fokker-Planck equation associated with (2.3):

$$
\frac{\partial}{\partial t} \rho_{t}=-\nabla \cdot\left(\rho_{t} b_{t}^{*}\right)-\Delta \rho_{t}
$$

and putting together the two equations one has:

$$
\frac{\partial}{\partial t} \rho_{t}=-\nabla \cdot\left(\rho_{t} v_{t}\right)
$$

where the definition of the current velocity has been used.

\section{The PATHWise FLUX ACROSS A BOUNDARY.}

Given an open set $D$ we want now to define the flux across $\partial D$ by the path of a diffusion.

In order to do this we would like to introduce a pathwise analogous of the flux as the function

$$
N_{\partial D}(\gamma):=N_{\partial D}^{+}(\gamma)-N_{\partial D}^{-}(\gamma),
$$

where $N_{\partial D}^{+}(\gamma)$ (resp. $N_{\partial D}^{-}(\gamma)$ ) denotes the number of inward (resp. outward) crossing by $[0,+\infty) \ni t \mapsto \gamma(t)$ of $\partial D$. The problem is that the above definition makes no sense since $\mathbb{P}$-a.s. the set $\left\{t: X_{t} \in \partial D\right\}$ has no isolated points and is uncountable. Therefore we are forced to proceed in an alternative way.

Let us observe that if $\#\{t: \gamma(t) \in \partial D\}<+\infty$ then $N_{\partial D}(\gamma)$ is the total mass of the random signed measure

$$
\sum_{t \in\{s: \gamma(s) \in \partial D\}} c(t) \delta_{t},
$$

where $c(t)=+1$ if $t$ corresponds to an outward crossing and $c(t)=-1$ if $t$ corresponds to an inward crossing. Therefore

$$
\sum_{t \in\{s: \gamma(s) \in \partial D\}} c(t) \delta_{t}=-\frac{d}{d t} \chi_{D}(\gamma(t)),
$$

where the derivative has to be intended in distributional sense, and thus we give the following

Definition 3.1. Given any open domain $D$, we define the random distribution

$$
\mu_{D}: \Omega \rightarrow \mathcal{D}^{\prime}(\mathbb{R})
$$


by

$$
\left\langle\mu_{D}(\gamma), \phi\right\rangle:=\chi_{D}(\gamma(0)) \phi(0)+\int_{0}^{+\infty} \chi_{D}(\gamma(t)) \dot{\phi}(t) d t
$$

where $\phi \in \mathcal{D}(\mathbb{R}) \equiv C_{c}^{\infty}(\mathbb{R})$.

Supposing now that $D$ is bounded and that $X_{t}$ is transient, we have that $\mathbb{P}$-almost surely the random distribution $\mu_{D}$ has a compact support and so its mass, which we denote by $N_{\partial D}$, is well defined. We define then the flux across $\partial D$ by $\Phi_{\partial D}:=\mathbb{E}\left(N_{\partial D}\right)$.

By the continuity equation (2.5) such an expectation can be explicitly calculated in terms of the current velocity $v$ (use [13], theorem 7):

Theorem 3.2. Let $\left(\Omega, \mathcal{F}, \mathbb{P}_{t}, X_{t}, \mathbb{P}\right)$ satify $H 1$ and $H$ 2, with $\rho_{t} \in H^{1}\left(\mathbb{R}^{3}\right)$ and $v_{t} \in H^{1}\left(\mathbb{R}^{3}\right)$ for any $t \geq 0$. For any open bounded domain $D$, with $\partial D$ a finite union of $C^{1}$ manifolds, one has

$$
\Phi_{\partial D}=\int_{0}^{+\infty} \int_{\partial D} \rho(t, x) v(t, x) \cdot n(x) d \sigma(x) d t,
$$

where $n$ denotes the outward unit normal vector along $\partial D$ and $\sigma$ is the surface measure.

\section{Diffusion With An ASYmptotic velocity.}

Since our goal is to define an asymptotic flux across hypersurfaces, we need to impose a condition on the time evolution of the process $\frac{1}{t} X_{t}$.

Definition 4.1. We say that the diffusion paths admit an asymptotic velocity when

H3)

$$
\lim _{t \uparrow \infty} \frac{1}{t} X_{t}=v_{\infty} \neq 0 \quad \mathbb{P} \text {-a.s. }
$$

and moreover $\mu_{\infty} \ll \lambda$, where $\lambda$ denotes the Lebesgue measure on $\mathbb{R}^{d}$ and $\mu_{\infty}$ is the distribution of $v_{\infty}$.

From now on by an open cone $C_{\Sigma}$ we will mean a set of the form

$$
\left\{\lambda x \in \mathbb{R}^{3}: x \in \Sigma, \lambda>0\right\},
$$

where $\Sigma$ is an open subset of the unit sphere with $\partial \Sigma$ a finite union of $C^{1}$ manifolds.

Remark 4.2. For any open cone $C_{\Sigma}$, any ball $B_{R}$ of radius $R$, and for any diffusion $\left(\Omega, \mathcal{F}, \mathbb{P}_{t}, X_{t}, \mathbb{P}\right)$ satifying $\mathrm{H} 1, \mathrm{H} 2$ and $\mathrm{H} 3$, one has

$$
\lim _{t \uparrow \infty} \chi_{C_{\Sigma} \cap B_{R}^{c}}\left(X_{t}\right)=\lim _{t \uparrow \infty} \chi_{C_{\Sigma}}\left(X_{t}\right)=\chi_{C_{\Sigma}}\left(v_{\infty}\right) \quad \mathbb{P} \text {-a.s. }
$$


See [13 for a two-line proof. Thus hypotheses H3 requires that the limiting velocity is non negligible and such that asymptotically the paths have the same direction as their limiting velocity. For example the Brownian motion in $\mathbb{R}^{3}$ is transient but it has no limiting velocity according to our definition because of the S.L.L.N. Only a Brownian motion with drift could satisfy the requirement of our definition.

A simple but general condition giving the existence of a limiting velocity, which is again a finite relative entropy condition (now on the full $\sigma$-algebra and with respect to $\widetilde{\mathbb{P}}$, see the proof below), is given in the following (see [3]):

Theorem 4.3. Let $\left(\Omega, \mathcal{F}, \mathbb{P}_{t}, X_{t}, \mathbb{P}\right)$ satify H1 and H2. If moreover one has:

$$
\mathbb{E} \int_{t_{0}}^{+\infty}\left\|b\left(t, X_{t}\right)-X_{t} / t\right\|^{2} d t<+\infty \quad t_{0}>0
$$

then:

$$
\lim _{t \uparrow \infty} \frac{1}{t} X_{t}=v_{\infty} \quad \mathbb{P}-\text { a.s. }
$$

for some random variable $v_{\infty}$.

Proof. The condition (4.1) implies, by [5], prop. 2.11, that $\mathbb{P} \ll \widetilde{\mathbb{P}}$ on $\sigma\left(X_{s}, t_{0} \leq s<+\infty\right)$, where $\widetilde{\mathbb{P}}$ is the weak solution of the simple stochastic differential equation

$$
d X_{t}=\frac{1}{t} X_{t} d t+d \widetilde{W}_{t}
$$

with $\widetilde{W}_{t}$ a standard Wiener process and is such that $\mathbb{P} \circ X_{t_{0}}^{-1}=\widetilde{\mathbb{P}} \circ X_{t_{0}}^{-1}$. Therefore:

$$
d\left(\frac{1}{t} X_{t}\right)=-\frac{1}{t^{2}} X_{t} d t+\frac{1}{t} d X_{t}=\frac{1}{t} \widetilde{W}_{t}
$$

and so

$$
\frac{1}{t} X_{t}=\frac{1}{t_{0}} X_{t_{0}}+\int_{t_{0}}^{t} \frac{1}{s} d \widetilde{W}_{s}
$$

Since

$$
\widetilde{\mathbb{E}}\left(\int_{t_{0}}^{+\infty} \frac{1}{s} d \widetilde{W_{s}}\right)^{2}=\int_{t_{0}}^{+\infty} \frac{1}{s^{2}} d s<+\infty
$$

by Doob's martingale convergence theorem one gets $\widetilde{\mathbb{P}}$-a.s. convergence of $\frac{1}{t} X_{t}$. The theorem then follows by absolutely continuity. 
Remark 4.4. Under the same hypotheses of Theorem 4.3 it is possible to prove (see [3]) that the random variable $v_{\infty}$ generates the tail $\sigma-$ algebra

$$
\mathcal{T}:=\bigcap_{t>0} \sigma\left(X_{s}, s \geq t\right)
$$

\section{The Asymptotic FluX ACROSS HypersurfaCes.}

Let us consider hypersurfaces of the following type:

$$
\Sigma_{R}=C_{\Sigma} \cap S_{R}
$$

where $S_{R}$ is the sphere of radius $R$. We will define the pathwise flux across $\Sigma_{R}$ in the limit when $R \uparrow \infty$.

Suppose at first that $\#\left\{t: \gamma(t) \in \Sigma_{R}\right\}<+\infty$. Since, by H3, $t \mapsto$ $\gamma(t)$ is definitively either in $C_{\Sigma}$ or in $\bar{C}_{\Sigma}^{c}$, if $R$ is sufficiently large one has

$$
\sum_{t \in\left\{s: \gamma(s) \in \Sigma_{R}\right\}} c(t) \delta_{t}=\sum_{t \in\left\{s: \gamma(s) \in \Sigma_{R} \cup\left(\partial C_{\Sigma} \cup B_{R}^{c}\right)\right\}} c(t) \delta_{t}=\frac{d}{d t} \chi_{C_{\Sigma} \cap B_{R}^{c}}(\gamma(t)) .
$$

We are therefore lead to give the following

Definition 5.1. The asymptotic pathwise flux across $\Sigma$ is defined by

$$
N_{\Sigma}^{a}:=\lim _{R \uparrow \infty} N_{\Sigma_{R}}
$$

where $N_{\Sigma_{R}}$ is the total mass of the random distribution $-\mu_{C_{\Sigma} \cap B_{R}^{c}}$.

The following result shows that the above definition makes sense.

Theorem 5.2. Let $\left(\Omega, \mathcal{F}, \mathbb{P}_{t}, X_{t}, \mathbb{P}\right)$ satify H1, H2 and H3. Then $\mathbb{P}$ almost surely the random distribution $\mu_{C_{\Sigma} \cap B_{R}^{c}}$ has a compact support and so its mass $N_{\Sigma_{R}}$ is well defined. Moreover one has

$$
\lim _{R \uparrow \infty} N_{\Sigma_{R}}=\chi_{C_{\Sigma}}\left(v_{\infty}\right) \quad \mathbb{P}-\text { a.s. } .
$$

Proof. Let

$$
\tau_{R}(\gamma):=\sup \{t \geq 0:\|\gamma(t)\|<R\} .
$$

By H3, $\tau_{R}<+\infty \mathbb{P}$-a.s. Thus $\mu_{C \cap B_{R}^{c}}$ has compact support $\mathbb{P}-$ a.s..

Let $\phi_{\gamma} \in \mathcal{D}(\mathbb{R})$ such that $\phi_{\gamma}=1$ on a neighbourhood of $\left[0, \tau_{R}(\gamma)\right]$. By the definition of $\mu_{C_{\Sigma} \cap B_{R}^{c}}$ one has

$$
\begin{aligned}
\left\langle\mu_{C_{\Sigma} \cap B_{R}^{c}}(\gamma), \phi_{\gamma}\right\rangle & =-\chi_{C_{\Sigma} \cap B_{R}^{c}}(\gamma(0))-\chi_{C_{\Sigma}}\left(v_{\infty}(\gamma)\right) \int_{\tau_{R}(\gamma)}^{+\infty} \dot{\phi}_{\gamma}(t) d t \\
& =-\chi_{C_{\Sigma} \cap B_{R}^{c}}(\gamma(0))+\chi_{C_{\Sigma}}\left(v_{\infty}(\gamma)\right)
\end{aligned}
$$

and the thesis then immediately follows by taking the limit $R \uparrow \infty$. 
The next theorem shows that the definition of asymptotic flux across $\Sigma$ by $\Phi_{\Sigma}^{a}:=\mathbb{E}\left(N_{\Sigma}^{a}\right)$ is consistent with the result given in theorem 3.2 in the case of the flux across a boundary:

Theorem 5.3. Let $\left(\Omega, \mathcal{F}, \mathbb{P}_{t}, X_{t}, \mathbb{P}\right)$ satify H1, H2 and H3 and suppose $\rho_{t} \in H^{1}\left(\mathbb{R}^{3}\right), v_{t} \in H^{1}\left(\mathbb{R}^{3}\right)$ for any $t \geq 0$ and

$$
\mathbb{E} \int_{0}^{T}\left\|\nabla v\left(t, X_{t}\right)\right\|^{2} d t<+\infty
$$

for some $T>0$. Then

$$
\Phi_{\Sigma}^{a} \equiv \mathbb{E}\left(\chi_{C_{\Sigma}}\left(v_{\infty}\right)\right)=\lim _{R \uparrow \infty} \int_{0}^{+\infty} \int_{\Sigma_{R}} \rho(t, x) v(t, x) \cdot n(x) d \sigma(x) d t .
$$

Proof. Proceeding as is 13 one has

$$
\mathbb{E}\left(\chi_{C_{\Sigma}}\left(v_{\infty}\right)\right)=\lim _{R \uparrow \infty} \int_{0}^{+\infty} \int_{\Sigma_{R} \cup\left(\partial C_{\Sigma} \cap B_{R}^{c}\right)} \rho(t, x) v(t, x) \cdot n(x) d \sigma(x) d t
$$

The proof is then concluded by proving that:

$$
\lim _{R \uparrow \infty} \int_{0}^{+\infty} \int_{\partial C_{\Sigma} \cap B_{R}^{c}} \rho(t, x) v(t, x) \cdot n(x) d \sigma(x) d t=0
$$

By the monotone convergence theorem, (5.2) follows from

$$
\int_{0}^{T} \int_{\partial\left(C_{\Sigma} \cap B_{R}^{c}\right)}|\rho(t, x) v(t, x) \cdot n(x)| d \sigma(x) d t<+\infty
$$

for some $T>0$. Since

$$
\begin{aligned}
& \int_{0}^{T} \int_{\partial\left(C_{\Sigma} \cap B_{R}^{c}\right)}\left|\rho(t, x)^{1 / 2} \rho(t, x)^{1 / 2} v(t, x) \cdot n(x)\right| d \sigma(x) d t \\
\leq & \int_{0}^{T}\left(\left(\int_{\partial\left(C_{\Sigma} \cap B_{R}^{c}\right)} \rho(t, x) d \sigma(x)\right)^{1 / 2} \times\right. \\
& \left.\left.\left(\int_{\partial\left(C_{\Sigma} \cap B_{R}^{c}\right)} \rho(t, x)\right)\|v(t, x)\|^{2} d \sigma(x)\right)^{1 / 2}\right) d t,
\end{aligned}
$$


by trace estimates on functions in $H^{1}\left(\mathbb{R}^{3}\right)$ (see e.g. [1], chap.5) one has

$$
\begin{aligned}
& \int_{0}^{T} \int_{\partial\left(C_{\Sigma} \cap B_{R}^{c}\right)}|\rho(t, x) v(t, x) \cdot n(x)| d \sigma(x) d t \\
& \leq \int_{0}^{T}\left(\int_{\mathbb{R}^{3}}\left(\rho(t, x) d x+\int_{\mathbb{R}^{3}}\left\|\nabla \rho^{1 / 2}(t, x)\right\|^{2} d x\right)^{1 / 2} \times\right. \\
& \left.\times\left(\int_{\mathbb{R}^{3}} \rho(t, x)\|v(t, x)\|^{2} d x+\int_{\mathbb{R}^{3}}\left\|\nabla\left(\rho^{1 / 2} v\right)(t, x)\right\|^{2} d x\right)^{1 / 2}\right) d t .
\end{aligned}
$$

From $(2.4)$ we have $u=\frac{\nabla \rho}{2 \rho}$, hence one has $\nabla \rho^{1 / 2}=u \rho^{1 / 2}$ and $\nabla\left(\rho^{1 / 2} v\right)=$ $u v \rho^{1 / 2}+\rho^{1 / 2} \nabla v$. Therefore in order to obtain (5.3) it is sufficient to have (5.1) and

$$
\mathbb{E} \int_{0}^{T}\left(\left\|u\left(t, X_{t}\right)\right\|^{2}+\left\|v\left(t, X_{t}\right)\right\|^{2}\right) d t<+\infty
$$

which is equivalent to $(2.2)$.

\section{CONCLUSION}

The present paper introduces the notion of flux across the boundary of a bounded open set $D$ in Euclidean space. For regular compact domains it is the expectation of the total mass of the (almost surely compactly supported) random distribution given by the distributional time-derivative of the functional

$$
-\chi_{D}\left(X_{t}\right)
$$

where the diffusion $X_{t}$, which satisfies the stochastic differential equation $d X_{t}=b_{t}\left(X_{t}\right) d t+d W_{t}$, is transient. If, moreover, the limit

$$
\lim _{t \uparrow \infty} \frac{1}{t} X_{t}
$$

exists almost surely, then to the notion of asymptotic flux is given a sense as well. The flux can be expressed in terms of $\rho_{t}$, the density of the distribution of $X_{t}$, and the current velocity $v_{t}=b_{t}-\frac{1}{2} \nabla \log \rho_{t}$.

\section{REFERENCES}

[1] Burenkov, V.I.: Sobolev Spaces on Domains. Stuttgart, Leipzig: Teubner 1998

[2] Carlen, E.: Conservative Diffusions. Commun. Math. Phys. 94, 293-315 (1984)

[3] Carlen, E.: The Pathwise Description of Quantum Scattering in Stochastic Mechanics. Lecture Notes in Physics 262, 139-147. Berlin, Heidelberg, New York: Springer-Verlag 1986 
[4] Carmona, R.: Probabilistic Construction of Nelson Processes. Probabilistic Methods in Mathematical Physics (Katata/Kyoto 1985), 55-81. Boston: Academic Press 1987

[5] Ershov, M.: On the Absolute Continuity of Measures Corresponding to Diffusion Processes. Theory of Prob. and Appl. 17, 169-174 (1972)

[6] Föllmer, H.: Time Reversal on Wiener Space. Lecture Notes in Mathematics 1158, 119-129. Berlin, Heidelberg, New York: Springer-Verlag 1986

[7] Föllmer, H. and Wakolbinger, A.: Time Reversal of Infinite-dimensional Diffusions. Stochastic Process. Appl. 22, 59-77 (1986)

[8] Haussmann, U and Pardoux, E.: Time Reversal of Diffusions. Ann. Probab. 14, 1188-1205 (1986)

[9] Millet, A., Nualart, D. and Sanz, M.: Integration by Parts and Time Reversal of Diffusion Processes. Ann. Probab. 17, 208-238, (1989)

[10] Nelson, E.: Derivation of the Schrödinger Equation from Newtonian Mechanics. Phys. Rev. 150, 1079-1085 (1966)

[11] Nelson, E.: Dynamical Theories of Brownian Motion. Princeton: Princeton Univ. Press 1967

[12] Nelson, E.: Quantum Fluctuations. Princeton: Princeton Univ. Press 1985

[13] Posilicano, A. and Ugolini, S.: Scattering into Cones and Flux across Surfaces in Quantum Mechanics: a Pathwise Probabilistic Approach. J. Math. Phys. 43, 5386-5399 (2002)

[14] Zheng, W. and Meyer, P.A.: Quelques résultats de Mécanique Stochastique. Lecture Notes in Mathematics 1059, 223-244. Berlin, Heidelberg, New York: Springer-Verlag 1985

Dipartimento di Scienze, Università dell'Insubria, via VallegGio 11, I-22100 COMO, ITALY

E-mail address: andreap@uninsubria.it

Dipartimento di Matematica, Università di Matematica, via Saldini 50, I-20133 Milano, Italy

E-mail address: ugolini@mat.unimi.it 